\title{
Absorption spectroscopy of quantum black holes with gravitational waves
}

\author{
Ivan Agullo \\ Department of Physics and Astronomy, Louisiana State University, Baton Rouge, LA 70803-4001, \\ USA \\ Vitor Cardoso \\ CENTRA, Departamento de Física, Instituto Superior Técnico - IST, Universidade de Lisboa - UL, \\ Avenida Rovisco Pais 1, 1049 Lisboa, Portugal \\ Adrián del Rio \\ Institute for Gravitation and the Cosmos, Physics Department, Penn State, University Park, PA \\ 16802-6300, USA. \\ Michele Maggiore \\ Departement de Physique Theorique and Center for Astroparticle Physics, Universite de Geneve, 24 \\ quai Ansermet, CH-1211 Geneve 4, Switzerland \\ Jorge Pullin \\ Department of Physics and Astronomy,Louisiana State University, Baton Rouge, LA 70803-4001, \\ USA
}

\begin{abstract}
The observation of electromagnetic radiation emitted or absorbed by matter was instrumental in revealing the quantum properties of atoms and molecules in the early XX century, and constituted a turning-point in the development of the quantum theory. Quantum mechanics changes dramatically the way radiation and matter interact, making the probability of emission and absorption of light strongly frequency dependent, as clearly manifested in atomic spectra. In this essay, we advocate that gravitational radiation can play, for the quantum aspects of black holes, a similar role as electromagnetic radiation did for atoms, and that the advent of gravitational-wave astronomy can bring this fascinating possibility to the realm of observations.
\end{abstract}

Essay written for the Gravity Research Foundation 2021 Awards for Essays on Gravitation

Submitted on March 30, 2021 
That black holes (BHs) have similarities with atoms was emphasized by Bekenstein five decades ago [1, 2]. Not only their physical states are characterized by a few numbers - mass $M$, spin $J$ and possibly electric charge- but these parameters may only take discrete values if one applies well established arguments by Bohr, Sommerfeld, Ehrenfest and others. Indeed, based on the observation that the area of BHs is an adiabatic invariant in general relativity (GR), Bekenstein concluded that the horizon area should be quantized, and further argued that the corresponding spectrum must be equally spaced in units of a fundamental quantum $\Delta A=\alpha \ell_{P l}^{2}$, of the order of the Planck area $\ell_{P l}^{2}=\frac{\hbar G}{c^{3}} \approx 10^{-70} \mathrm{~m}^{2}$, where $\alpha$ is a constant of order one. Upon standard quantization of angular momentum, one concludes that the mass or energy spectrum must be discrete. This quantization changes drastically the way BHs interact with classical radiation, discretizing the frequency of the waves they can absorb or emit [3], in analogy to the atomic case. Bekenstein and Mukhanov [4] explored potential consequences for the emission spectrum of BHs, i.e., for spontaneous Hawking radiation. The low Hawking temperature makes the observation of the predicted effects unreachable, at least for astrophysical BHs. However, the birth of GW astronomy offers an interesting alternative: to study the consequences of $\mathrm{BH}$ area quantization for the absorption spectrum. This is the central topic of this essay.

An obvious question is the following: why should a discretization at the Planck scale of an astrophysical BH horizon affect the low-frequency GWs that we observe in our detectors? The frequencies $\omega_{\text {abs }}$ - or energies - that BHs can absorb are determined by their mass spectrum. In GR, the transition between two close states of definite mass is determined by the first law of $\mathrm{BH}$ mechanics [5]:

$$
\omega_{\mathrm{abs}} \equiv \frac{c^{2} \Delta M}{\hbar}=\frac{\kappa c^{2}}{8 \pi G} \frac{\Delta A}{\hbar}+\Omega_{H} \frac{\Delta J}{\hbar},
$$

where $\kappa$ denotes the surface gravity and $\Omega_{H}$ the horizon angular velocity. Since both $\kappa$ and $\Omega_{H}$ scale as $M^{-1}$, Eq. (1) reveals that $\Delta M \propto \frac{1}{M}$ : the energy levels of a BH are not equally spaced when $A$ and $J$ are both uniformly discretized. Hence, energy levels get closer together for heavier BHs. For instance, the basic absorption frequency of a non-rotating $\mathrm{BH}$ is $\omega_{\mathrm{abs}}=\frac{c^{3}}{32 \pi G M_{\odot}} \alpha \frac{M_{\odot}}{M}$, where we have expressed $M$ in units of the solar mass $M_{\odot}$. The interesting observation is that the combination of constants $\frac{c^{3}}{32 \pi G M_{\odot}}$ is approximately $2 \mathrm{kHz}$ - this is not a numerical accident: parametrically this is the same as the frequencies of the quasi-normal modes (QNMs) discussed below. Thus, the large mass of typical astrophysical BHs is responsible for translating Planck-scale discretization of the $\mathrm{BH}$ area to frequencies within the window of $\mathrm{GW}$ detectors. 
Recent investigations have revealed two observable channels in the coalescence of binary black hole systems that can inform us on the discrete energy spectrum of BHs, related to the ringdown and the inspiral phases [6, 7, 8].

The ringdown phase is accurately described by a perturbed Kerr BH, which dissipates its perturbations via its characteristic QNMs. Most of the energy carried by the QNMs moves outwards to GW detectors, but a non-negligible fraction propagates inwards, towards the horizon. This is a remarkably monochromatic radiation, because it is dominated by the quadrupolar mode $\ell=2, m=2$ and the fundamental tone $n=0$. The oscillation frequency of this wave is $M \operatorname{Re} \omega_{022} \simeq 1.5251-1.1568(1-a)^{0.1292}$, where $a=J / M^{2}$. If this frequency does not match any transition of the $\mathrm{BH}$ mass spectrum, Eq. (1), the probability of absorption will be suppressed. The incoming radiation will then start propagating outwards. Once it interacts with the light ring, a portion will be scattered back, and the process will repeat in time. As a result, an external observer will see an initial GW burst followed by a set of echoes with increasingly smaller amplitudes [9, 7, 10]. The values of these amplitudes depend on the exact absorption properties of the $\mathrm{BH}$ - determined by unknown microscopic physics - and on the transmissivity of the potential barrier. Reasonable estimates indicate that the amplitude of the first echo could be as large as a percent of the initial GW front. The ability to detect these echoes depends on our capacity to produce faithful templates. For existing phenomenological families of echo waveforms, Bayesian analysis in the LIGO/Virgo data do not find evidence for echoes with amplitude $0.1-0.2$ times to the original signal peak [11]. Observational constraints will improve significantly in the near future with 3G detectors such as the Einstein Telescope (ET) [12] and the space mission LISA [13].

The second possibility to test the area quantization hypothesis relies on the inspiral. During this stage the system emits GW radiation, again dominated by the quadrupolar mode, with frequency given by the binary orbital angular velocity $\Omega, \omega \approx 2 \Omega$. Classically, the individual BHs absorb a portion of these waves, which induce tidal forces that distort the horizons. But as the distorted BHs rotate, energy is dissipated gravitationally, and transferred to the inspiral dynamics. This phenomenon is known as tidal heating [7]. Now, because the orbital angular velocity is considerably low during inspiral, the frequency of these GWs is smaller than the lowest absorption frequency of the individual BHs, $\omega_{\text {abs }} \sim 2 \Omega_{H}$ (see Eq. (1) and comments below). Consequently, the GW absorption is expected to be suppressed - this is analog to the familiar frequency threshold in the photoelectric effect-modifying the orbital evolution of the binary as compared to the classical prediction. One can study this effect by introducing an absorption parameter $\gamma$ in the waveform (multiplying the $2.5 \mathrm{PN} \times \log v$ GR term, [6]). 
Quantization of area decreases $\gamma$ relative to its value for classical BHs, $\gamma_{\text {class }}=1$. The analysis of [6] reveals that advanced detectors such as LISA and the ET will reach the desired sensitivity to discriminate among these values of $\gamma$.

Although these effects are consequences of the quantization of the $\mathrm{BH}$ energies, this discreteness alone is not sufficient. The energy levels are determined from the area and angular momentum quantum numbers $n$ and $j$ by $M_{n, j}=\sqrt{\hbar} \sqrt{\frac{\alpha n}{16 \pi}+\frac{4 \pi j^{2}}{\alpha n}}$. This spectrum consists not only of Schwarzchild states, but it also includes all spinning configurations. It is a highly irregular and crowded spectrum which, under consideration of the linewidths (see below), approaches a continuum. Nevertheless, as recently pointed out in [8], conservation of angular momentum introduces constraints, or "selection rules", which, as in atomic physics, restrict the energy levels that the BH can transition to when it interacts with a GW mode $(\omega, \ell, m)$. As previously emphasized, the GWs generated during the inspiral and ringdown stages are dominated by quadrupolar modes $(\ell=2, m=2)$, that single out BH energy levels differing by $\Delta j=2$. These levels form a small and simple subset of the spectrum $M_{n, j}$. Furthermore, since these GWs are also highly monochromatic, unless their frequency matches one of the absorption frequencies of the $\mathrm{BH}$, the probability of absorption would be suppressed, giving rise to the effects described above. Therefore, it is the combination of energy quantization and angular momentum conservation that gives rise to observable effects.

Another important point to take into account is the width $\Gamma$ of the energy levels: observable effects exist only if $\Gamma$ does not make the relevant energy levels discussed above to overlap [14, 8]. This linewidth can be estimated as $\Gamma=\hbar / \tau$, where $\tau$ is the spontaneous decay rate, i.e., the timescale associated with Hawking emission. This can be computed following Page's calculations [15]. Reference [8] has computed the ratio $R(a, \alpha)=\Gamma /\left(\left.\hbar w_{\mathrm{abs}}\right|_{\Delta j=2}\right)$, and has showed it is a function of the $\mathrm{BH}$ spin $a$ and the size $\alpha$ of the fundamental area gap; the explicit dependence on $\mathrm{BH}$ mass $M$ cancels out. $R(a, \alpha)$ can be large if $\alpha$ is sufficiently small - in fact $R$ diverges in the limit $\alpha \rightarrow 0$, in which the $\mathrm{BH}$ recovers the continuous energy spectrum. The interesting quantity is therefore $\alpha_{\text {crit }}(a)$, the value of $\alpha$ below which the relevant energy levels in a binary coalescence overlap, $R\left(a, \alpha_{\text {crit }}(a)\right)=1$. This quantity is

$$
\alpha_{\text {crit }}(a)=0.0842+0.2605 a^{2}+0.0320 e^{5.3422 a^{3}}
$$

accurate to within $2 \%$ for $a<0.9$. As an example, for $a \approx 0.7$ - the spin of the remnant $\mathrm{BH}$ found in a large fraction of the observed mergers - one obtains $\alpha_{\text {crit }}=0.42$. This is one order of magnitude below $4 \log 2$, the smallest value of $\alpha$ that has been discussed 
in the literature. Hence, for reasonable values of $\alpha$ and $\mathrm{BH}$ spins, the relevant energy levels do not overlap.

A summary of this discussion is represented in Fig. 1] (see [8] for more details). The spin of BH's enriches the phenomenology in an unforeseen manner. In particular, the analysis shows that an observation of echoes for binary mergers sampling a large enough range of the $\mathrm{BH}$ spin $a$, provides a way to test the $\mathrm{BH}$ area spectrum and to determine the fundamental constant $\alpha$.

As surprising as it may sound, that GW radiation can inform us about quantum aspects of BHs is a plausible possibility, which certainly deserves further scrutiny. We encourage the quantum gravity community to derive concrete predictions, including GWs templates, which could be contrasted with current and future GW observations.

Acknowledgments. V. C. acknowledges financial support provided under the European Union's H2020 ERC Consolidator Grant "Matter and strong-field gravity: New frontiers in Einstein's theory" grant agreement no. MaGRaTh-646597. This project has received funding from the European Union's Horizon 2020 research and innovation programme under the Marie Sklodowska-Curie grant agreement No 690904. We thank FCT for financial support through Project No. UIDB/00099/2020 and through grant PTDC/MAT-APL/30043/2017. The authors acknowledge networking support by the GWverse COST Action CA16104, "Black holes, gravitational waves and fundamental physics." AdR. acknowledges support under NSF grant PHY-1806356 and the Eberly Chair funds of Penn State. MM is supported by the Swiss National Science Foundation and by the SwissMap National Center for Competence in Research. IA is supported by the NSF CAREER grant PHY-1552603 and by the Hearne Institute for Theoretical Physics. JP is supported by grant NSF-1903799, by the Hearne Institute for Theoretical Physics and CCT-LSU.

\section{References}

[1] J. D. Bekenstein, "The quantum mass spectrum of the Kerr black hole," Lett. Nuovo Cim. 11 (1974) 467.

[2] J. D. Bekenstein, "Quantum black holes as atoms," in 8th Marcel Grossmann Meeting on Recent Developments in Theoretical and Experimental General Relativity, Gravitation and Relativistic Field Theories (MG 8), pp. 92-111. 6, 1997. arXiv:gr-qc/9710076. 


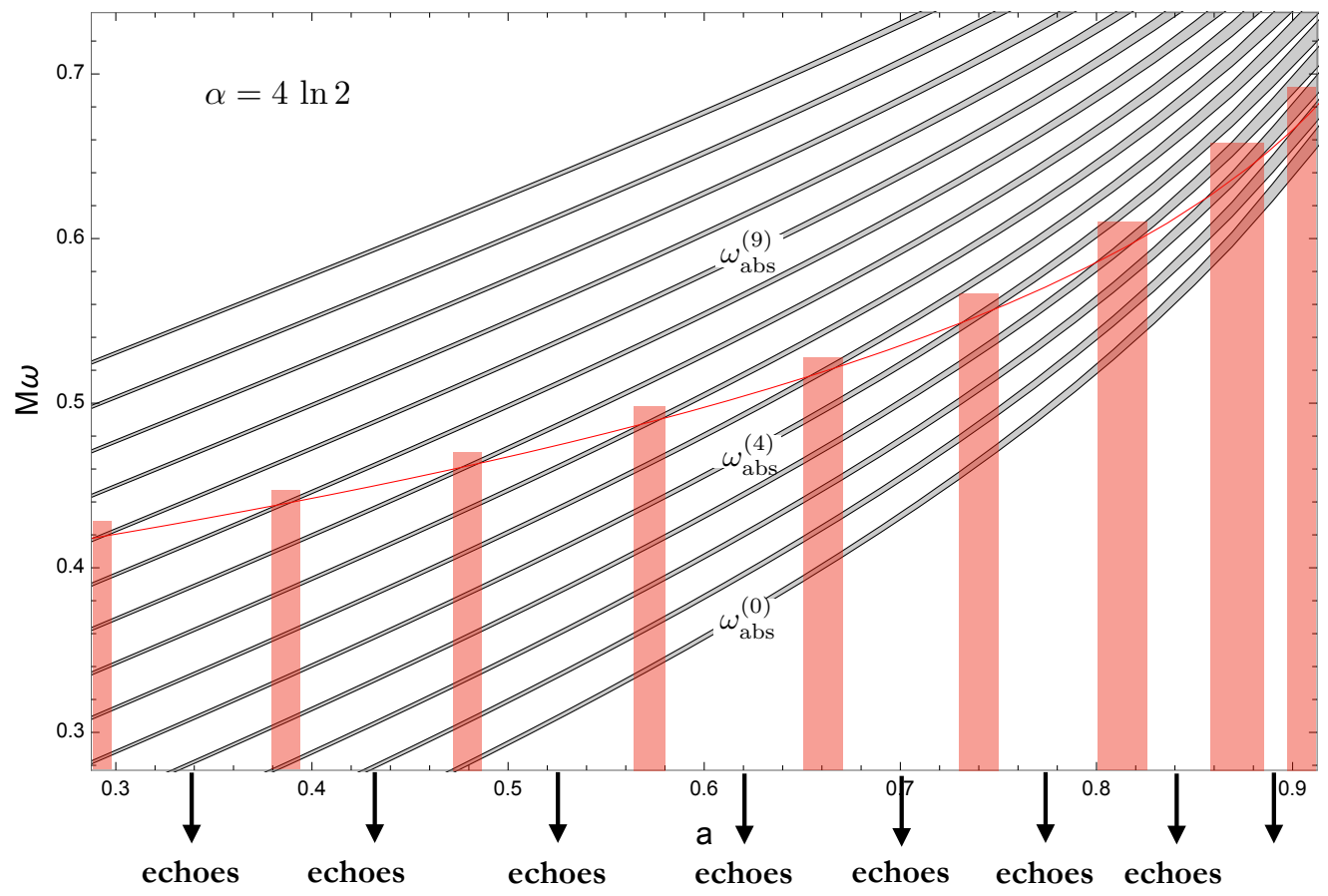

Figure 1: Black lines: absorption frequencies associated with the relevant energy levels of BHs in a binary merger. The (gray) thickness of the black lines measures the width $\Gamma$ of the spectral lines. The red line is the oscillation frequency of the dominant QNM, as a function of the BH spin $a$. This plot does not change with the mass $M$ of the final black hole, since all quantities plotted scale in the same manner. The intersection of the red and black lines, highlighted with vertical red bands, correspond to values of $a$ for which the probability of absorption of the dominant QNM is close to one. Echoes are therefore expected in the regions in between intersections. This plot is obtained using the smaller value of $\alpha$ that has been discussed in the literature. Larger values of $\alpha$ increase the range of $a$ for which echoes are expected.

[3] V. F. Foit and M. Kleban, "Testing quantum black holes with gravitational waves," Classical and Quantum Gravity 36 no. 3, (2019) 035006.

[4] J. D. Bekenstein and V. F. Mukhanov, "Spectroscopy of the quantum black hole," Phys. Lett. B 360 (1995) 7-12, arXiv:gr-qc/9505012.

[5] J. M. Bardeen, B. Carter, and S. W. Hawking, "The Four laws of black hole mechanics," Commun. Math. Phys. 31 (1973) 161-170.

[6] A. Maselli, P. Pani, V. Cardoso, T. Abdelsalhin, L. Gualtieri, and V. Ferrari, "Probing Planckian corrections at the horizon scale with LISA binaries," Phys. 
Rev. Lett. 120 no. 8, (2018) 081101, arXiv:1703.10612 [gr-qc].

[7] V. Cardoso, V. F. Foit, and M. Kleban, "Gravitational wave echoes from black hole area quantization," JCAP 08 (2019) 006.

[8] I. Agullo, V. Cardoso, A. del Rio, M. Maggiore, and J. Pullin, "Potential Gravitational Wave Signatures of Quantum Gravity," Phys. Rev. Lett. 126 no. 4, (2021) 041302, arXiv:2007.13761 [gr-qc].

[9] V. Cardoso, E. Franzin, and P. Pani, "Is the gravitational-wave ringdown a probe of the event horizon?," Phys. Rev. Lett. 116 no. 17, (2016) 171101, arXiv:1602.07309 [gr-qc]. [Erratum: Phys.Rev.Lett. 117, 089902 (2016)].

[10] V. Cardoso and P. Pani, "Testing the nature of dark compact objects: a status report," Living Rev. Rel. 22 no. 1, (2019) 4, arXiv:1904.05363 [gr-qc].

[11] J. Westerweck, A. Nielsen, O. Fischer-Birnholtz, M. Cabero, C. Capano, T. Dent, B. Krishnan, G. Meadors, and A. H. Nitz, "Low significance of evidence for black hole echoes in gravitational wave data," Phys. Rev. D97 no. 12, (2018) 124037. arXiv:1712.09966 [gr-qc]

[12] M. Maggiore et al., "Science Case for the Einstein Telescope," JCAP 03 (2020) 050, arXiv: 1912.02622 [astro-ph.CO]

[13] LISA Collaboration, P. Amaro-Seoane et al., "Laser Interferometer Space Antenna," arXiv:1702.00786 [astro-ph.IM].

[14] A. Coates, S. H. Volkel, and K. D. Kokkotas, "Spectral Lines of Quantized, Spinning Black Holes and their Astrophysical Relevance," Phys. Rev. Lett. 123 no. 17, (2019) 171104, arXiv: 1909.01254 [gr-qc].

[15] D. N. Page, "Particle Emission Rates from a Black Hole. 2. Massless Particles from a Rotating Hole," Phys. Rev. D 14 (1976) 3260-3273. 